\title{
Living donor liver transplantation for huge polycystic liver disease with recipient liver splitting method: a case report
}

Hyun-Jeong Kim

Department of Surgery-Transplantation, Severance Hospital, Seoul, Korea

Background: Polycystic liver disease (PLD) can progress to massive hepatomegaly resulting in impaired performance status and quality of life. In PLD patients with diffuse liver cysts with few areas of normal parenchyma, liver transplantation (LT) can be the only curable treatment. But LT can be extremely challenging due to the massiveness and hardness of the native liver, which makes difficulty in mobilization and access to vascular structures. We report our case of LT for massive hepatomegaly due to symptomatic PLD.

Case report: A 53-year-old male was diagnosed with autosomal dominant polycystic kidney disease in 1998. After 11 years, he was diagnosed also with PLD. He was firstly listed for a kidney transplantation due to proceeded with end-stage renal disease in 2011. While waiting for deceased donor, abdominal discomfort aggravated due to huge size of kidney. So, he underwent bilateral nephrectomy sequentially. In July 2020, due to an enlargement of liver cysts and massive hepatomegaly, the patient developed severe clinical symptoms; abdominal discomfort, dyspepsia, poor oral intake. He decided to proceed with living donor LT first in February 2021. At LT, the graft mobilization was too hard not only because of the size, weight, and hardness of the organ, but also because of inflammation and adhesion due to previous several operations. After hilar dissection and all the vasculatures were ligated, the inferior vena cava was exposed and could be dissected up to right hepatic vein. However, the hepatic veins were not able to be identified due to the huge liver. Liver parenchyma was resected by anterior approach and each hepatic veins were isolated. And the recipient's liver was weighed $10,134 \mathrm{~g}$.

Conclusions: This case shows that even recipients with a massive liver weighing more than $10 \mathrm{~kg}$ can also undergo surgery successfully.

Corresponding author: Hyun-Jeong Kim

E-mail: HJKIM0110@yuhs.ac

(c) The Korean Society for Transplantation

This is an Open Access article distributed under the terms of the Creative Commons Attribution Non-Commercial License (http://creativecommons.org/licenses/by-nc/4.0/) which permits unrestricted non-commercial use, distribution, and reproduction in any medium, provided the original work is properly cited 